\title{
Insensitive Attitudes Encountered by Lesbian, Gay, Bisexual, (LGB) Older Adults in Healthcare Systems: Have We Made Wrong Choices?
}

\author{
Okpodi Julius $^{1 *}$ and Okhakhu Emmarex ${ }^{2}$ \\ ${ }^{1}$ Canadian Registered Social Worker, Canada \\ ${ }^{2}$ Canadian healthcare leadership, Canada
}

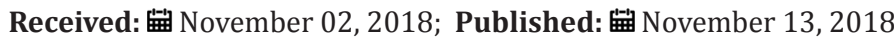

*Corresponding author: Okpodi Julius, Canadian Registered Social Worker, Canada

\begin{abstract}
Aging comes with life challenges. As older adults face changes in health, abilities, and social circumstances, their need for quality social and healthcare services grows. For the Lesbian, Gay, and Bisexual (LGB) older adults, their search for healthcare services unleashes extra burdens: the lack of respect, dignity from healthcare workers, so much that they fear treatment when navigating through the social and healthcare systems. This study referenced cultural sensitivity, honouring a relationship, and accommodating a partner as sensitive social support that requires immediate attention. The data gathered from this study were interpreted through the lens of thematic analysis. Thematic analysis was used to identify emerging themes. A theme captures something important about the data in relation to the research question and represents some level of patterned response or meaning within the data set.

While participants' narratives overlap and reinforce each other, thematic approach provides a way of understanding how LGB older adults construct meaning to their perceptions and experiences of services received within British Columbia Northern Health Authority boundaries. The findings from this study seek to identify perceived social inequalities (disrespect, homophobia, insensitive behaviours, and ill treatment) and help to improve human conditions by bridging the gaps that exist between social and healthcare services. Social and healthcare services discussed in this study specifically speak to housing and healthcare challenges faced by LGB older adults. Further, findings developed for this study allowed the understanding of social support for sexual minority older adults and the need for the field of gerontology to increase research on this population.
\end{abstract}

Keywords: LGB Older Adults; Homophobia; Cultural Sensitivity; Honoring LGB relationship; LGB Social Support; and Thematic Analysis.

\section{Introduction}

The most significant change occurring in Canada today is the growing number of older adults. The term "older adult" varies across disciplines. While gerontologists tend to define older adults as individuals who are 60 years of age or older, the federal government uses the ages 60-70 as a marker for the Canada Pension Plan (CPP) Canada Revenue Agency [1]. As people age, they continue to grow, gain knowledge, become active in their families, create and engage in healthy activities, or stay active in their communities. However, many LGB older adults referenced cultural sensitivity, honouring a relationship, and accommodating a partner as sensitive support that requires immediate attention.

LGB older adults have unique experiences and understandings of things. Work, marriage, relationships, social and life experiences, daily life routine-all these kinds of things matter and play a significant role Ghaziani [2]. Social and healthcare workers need to be culturally sensitive to LGB issues when brought to their attention. LGB issues and challenges need attention and should not be unaddressed and/or treated as a low priority. Against this background, the application of Leininger's culture care theory ensures LGB older adults' values, beliefs, and holistic care are factored into their treatment plan and/or overall social and healthcare needs Leininger [3]. Holistic care implies that the social and healthcare worker becomes culturally sensitive and trained, and can examine, acquire knowledge, interpret, and predict the phenomena in order to guide their practices. Cultural sensitivity may influence or help LGB older adults to improve their human conditions and address their social and healthcare needs Leininger [4]. 


\section{Theoretical Framework: Leininger's Culture Care Theory}

Leininger's cultural care theory Doornbos, Zandee, \& DeGroot [5] provided the framework for the exploration of the experiences and perceptions of LGB older adults in northern BC. This theoretical framework is based on an ethnonursing approach with the assumption that cultural, historical, and social factors are crucial for maintaining and stabilizing the well-being of an individual Doornbos [5], Leininger [6]. Leininger's theory outlined the following needs: to provide professional competency in services, to be sensitive to the client's culture, history, and other social factors Christensen [7] McFarland \& Wehbe-Alamah [8]. Leininger's theory pinpoints the variability in how people are treated within cultures. Further, the theory helps to highlight LGB as sexuality and gender identity-based culture and also helps to understand specifically the experiences of LGB as a culture or a subculture. Furthermore, Leininger's culture care theory lays the pathway for the richness that can be gained from a constructive face-to-face interaction with the participants. Such interaction increases trust, which lends credibility to the quality of the data Leininger [3]. This theoretical framework helps to understand LGB older adults' perceptions and experiences of growing older within northern, rural, and remote areas of British Columbia.

\section{Literature Review}

The most significant change occurring in Canada today is the growing number of older adults. This trend has shown the need to increase social support for older adults. Social support is a common area of study in aging research. Research has shown that LGB older adults have had unique life experiences growing up in an era that essentially thought their sexuality was either "abnormal" and required treatment and or illegal with a threat of incarceration. Amid all this, social support was characterized as a coping resource for LGB teens, young adults, and LGB adults in midlife and later Hunter [9]. The perception of social and emotional support has a greater and more positive impact on mental and physical health than the actual support received Hunter [9]. Social support has often been characterized as a function of the family of origin, and for older adults, their family of origin continues to provide most of their social support. The family as a societal construct has evolved to include blended families, inter-racial families, inter-religious families, same-sex headed families, and single-parent families. Rosenfeld [10] acknowledged that alternative family structures have always existed. However, researchers have often neglected these different family structures, and for these families, social support may look different than it did for previous cohorts.

Studies have shown that older LGB older adults are more likely to live alone and to have fewer children than their heterosexual counterparts Fredriksen-Goldsen [11], Gates [12]. On the other hand, research in social support has also offered positive findings. A study of social support networks of LGB older adults found that while many LGB older adults live alone, they have large social support networks Fredriksen-Goldsen \& Muraco [13]. Social support in the form of informal care (family members and friend caregivers and/or fictive kin) continues to support a positive aging experience for LGB older adults as they grow older in the north. An important aspect of social support networks of LGB older adults is the role of fictive kin. Fictive kin is a symbolic kinship used to describe created families Pinsker et al. [14]. Similar studies of the social support networks of LGB older adults have shown that fictive kin provide the highest level of social support, followed by life partners Taylor et al. [15].

\section{Lesbian, Gay, and Bisexual Subculture}

Lesbian, gay, and bisexual is a sexuality and gender identitybased culture or subculture, shared by the members of sexual minorities by virtue of their membership in those minorities Stone [16]. A subculture is defined as a set of people with distinct patterns of behaviours and beliefs that differentiate them from the larger culture of which they are a part Ghaziani [17], Taylor [15] \& Stone [16]. Subcultures are the basic unit of social interaction in which cultural innovation occurs. This social interaction gains wider acceptance as they are assimilated into the society Ghaziani, \& Baldassarri [17]. Furthermore, subcultures contain internal rule mechanisms (social rules or norms) that allow members to identify with one another and develop popular generational forms of identification that intersect with other markers of collective identity, such as race, gender, class, and sexuality Ghaziani \& Baldassarri [17]. Some characteristics of LGB subculture may include the gay pride movement organized to create awareness and acceptance and equal rights of LGB people; alternative lifestyles in mainstream television portrayed to promote LGB people as equivalent to heterosexuals; LGB dress, artwork, gay bars, and dance entertainment, just to mention a few. These were depicted to create LGB existence and demand for equal rights.

\section{Homophobic Challenges}

Despite this awareness, LGB older adults continue to witness covert discrimination and homophobic behaviours. Covert discrimination may refer to situations where provisions and practices put those of a particular sexual identity/gender identity at a disadvantage compared to others of a different sexual or gender identity Almeida et al. [18], Chance [19] While homophobia may be defined as anger towards, or fear of, LGB people, the concept in this context can also denote a mark of humiliation emanating from social ideologies about homosexuality Gallicano [20]. Homophobia and undesirable negative feelings about LGB are the roots of overt and covert discrimination and marginalization faced by some LGB people. Homophobia is shown in numerous ways, for instance in homophobic jokes, physical attacks, overt and covert discrimination, workplace harassment, and negative media representation, just to mention a few.

LGB older adults are prone to encounter intolerance, discrimination, harassment, and risk of violence owing to their 
sexual identity, unlike those that see themselves as heterosexual Stone [16]. However, Statistics Canada [21] shows that police reported hate crimes in Canada targeting sexual identity dropped $9 \%$ in 2015, from 155 cases in 2014 to 141 cases. Moral, religious, and political beliefs may be seen as some of the dynamic features that may strengthen homophobia on a larger scale. For example, a key social policy implication can highlight the provision of equal rights for LGB people (same-sex marriage, workplace policies, anti-harassment, etc.); this can absolutely influence attitudes and urge policy-makers to introduce legal structures, which may create a more enabling and inclusive society Cohen \& Ezer [22], HoyEllis [23], Ireland [24]. Homophobia is present everywhere, but LGB older adults experiencing homophobia in northern and rural Canada may have difficulties actualizing their hopes and needs Gallicano [20]. Living in a homophobic environment forces many LGB older adults to conceal their sexual identity for fear of the adverse and undesirable responses and consequences of coming out Rumens \& Broomfield [25].

\section{Psychological Distress}

LGB older adults cope effectively when they have the support of family and friends and participate with LGB groups or organizations and social networks Hoy-Ellis [23], Rumens \& Broomfield [25]. A significant number of LGB older adults have had to deal with stigmatization, marginalization, discrimination, and harassment without the availability of support systems. Some of them face pressure and tension resulting from high degrees of financial abuse, physical, emotional, and verbal attacks Hoy-Ellis [23]. All these have a negative impact on older adults' mental health and may lead to far-reaching levels of psychological anguish, self-harm, and suicide Hoy-Ellis [23], Stanley \& Duong [26]. Urban areas are more conducive to allowing LGB older adults to choose the aspects of their lives that they wish to disclose and to whom they wish to disclose their sexual identity, i.e., work, friends, family, neighbours, medical services, and associated activities. In remote communities, if LGB older adults decide to disclose their identity at work, suddenly the whole community and friends and family are aware of such disclosure Milner [27]. There is a greater likelihood that LGB older adults will experience psychological distress, physical disability, more anxiety, sadness, lower self-esteem, more loneliness, grief, and poorer mental health Stanley \& Duong [26] than their heterosexual counterparts Wallace et al. [28]. The next headings discuss the methods for this study. The next segment discusses the methodology used in this study.

\section{Research Method}

This qualitative exploratory research used a semi-structured, in-depth, face-to-face interview tool to gain an understanding of underlying reasons, experiences, opinions, and perceptions of the research participants Creswell [29]. This methodology helped to gain insights into participant's narratives and uncovered trends in their thoughts and opinions, and probed deeper into the phenomena
Bryman [30]. Furthermore, qualitative research is multimethod in focus, using an interpretive and naturalistic approach (qualitative researchers study things in their natural settings, attempt to make meaning of, and interpret the phenomena as presented by the participant). Qualitative research aims to understand the social reality or cultures as presented by study participants. Typically, qualitative research samples are small in size, and respondents are selected to fulfill a given quota Bryman [30], Creswell [29]. The indepth interview process was the primary data collection technique for gathering data in this qualitative study. Detailed data were gathered through open-ended questions that sometimes provide direct quotations, since such direct quotations help to articulate the questions and keep the response in perspective.

\section{The Ethics Approval Process}

Due to the sensitive nature of this study, the researchers conducted the research within the defined ethical parameter of competence: The researchers understood their limitations and did not assume to know more than they really do. Participants' demographic information was anonymized wherever possible. The choice of location outside of the normal work environment further protected their anonymity. The raw data was treated with utmost confidentiality. Privacy and confidentiality were thoroughly addressed on the consent form and verbally reiterated to the participants before the interview. Finally, there was no deception whatsoever in this research, whether in planning, language, data collection and analysis, or presentation of the findings.

\section{Thematic Analysis}

Thematic analysis is a broadly used qualitative analytic method Boyatzis [31]. This type of analysis is very inductive; i.e. the themes emerge from the data and are not imposed by the researcher. Thematic analysis is a qualitative analytic method that aims to uncover patterns or stories in data Braun \& Clarke [32]. Further, thematic analysis offers organization to the data analysis and helps the researcher to understand the potential of any issue more widely Meeks [33]. This thematic analysis was conducted over several stages. A set of codes was defined and each data item was labeled with one of these codes. The code schemes were checked to determine if they were balanced, repeatable, and unambiguous. The codes were refined and reviewed until the researchers reached a pre-determined agreement rate.

\section{Trustworthiness}

The value of qualitative research is determined by methodological considerations. Creswell [29] noted that the quality of qualitative research could be increased through rigorous methods and by building the credibility of the researchers. This qualitative research was conducted rigorously. We utilized several methods that supported research rigour. These methods included reflexive journaling, candidness, and feedback. Personal reflexivity is a significant aspect of this research. Also, we kept an audit trail 
and coding checks to ensure the trustworthiness of the study. Constructing meaning is learning and empowering Novak [34].

\section{Member Checking}

The member checking approach was used after data transcription and analysis to improve the accuracy, credibility, validate, and transferability in this study. Member checking is a form of triangulation that involved having those who were studied review the findings to offer a measure of accuracy Creswell [29]. After data transcription and initial analysis, the researchers gave participants both transcribed and analyzed sets of data to consider the findings critically and to comment on them. Participants were allotted two weeks to affirm that the summaries reflected their views, feelings, perceptions, and experiences or that they did not reflect these experiences. Also, participants were informed that failure to respond within the two weeks may imply the transcription and analysis accurately reflected their opinions. The findings in this research reviewed through the data analysis are presented below.

\section{Findings}

LGB participants shared their experiences and perceptions of cultural sensitivity, LGB relationships, and how to accommodate partners. LGB older adults were concerned and stated that these issues were unaddressed and/or treated as a low priority. Participants strongly believe that older adults, whether in heterosexual or homosexual relationships, need not be separated from their partners and loved ones, irrespective of their health conditions. As Beatrice discussed: They refused to take my partner's name as a next of kin. ... I had no immediate family except two underage children, and I did not want to give their names ... it came to the point where I was going to be refused admission if I didn't come up with a next of kin. So, I ended up giving my son's name and saying I had no contact for him. This was a challenging moment for Beatrice; she felt her autonomy was denied, and her relationship was not validated. Invalidation of same sex partners can cause loneliness, worthlessness, and sense of not belonging. Invalidation may be traumatizing for the LGB older adult who faces such a discriminatory act. Within the LGB community, next of kin could be same a sex partner, biological children, adopted children, friends, family/extended family members, church members, and even fictive kin who continue to support LGB older adults positively as they age successfully.

It is important for healthcare workers to create meaning of cultural safety for people. Next of kin may be required for record keeping and treatment purposes but should not be used to deny admission to a healthcare facility. Doris explained what honouring relationship means. She said: "we need to honour relationships. So, whether it is siblings or best friends, it does not matter. We need to create housing and services that allow those relationships to be fostered and continued." Doris felt that healthcare workers were very ethnocentric due to heterosexist (male and female) orientation Leininger [4]. Healthcare workers need to move faster along the trend of change, to accept, believe, and support LGB people seeking assistance. Validating LGB older adults' feelings, statements, and acknowledging/honouring their relationships, would foster well-being as they seek care and services. Doris wants healthcare workers to accept, honour, and respect a person's relationship: Healthcare institutions are currently separating partners and relationships, and best friends are forced to break bonds ... One thing I feel so strongly about is that people should not be separated from their partners; be it heterosexual or same sex, it doesn't matter. I have seen this in the past and I want it changed, and if you wish to separate them, give them options.

Doris's believes that institutions separate partners and relationships, and best friends are forced to break bonds. Doris believes that partners should not be separated, irrespective of the health challenges or sexual identity. If an older adult is sick and hospitalized in a facility, a healthcare worker should ask if there is anyone in that facility he/she wants to be paired with, be it a partner or best friend. It might be a room with separate bed space. The older adult should have the option to stay with a partner or best friend or stay in a separate facility. Doris believes that older adults have made a life commitment and would like to see each other and if possible hold hands even in their sick bed. She said, "This is comforting and healing for us." The ideas in a person's mind are very influential, and when an individual (Doris) is faced with certain experiences; she tends to relate those experiences with her previous views, constructing a better understanding out of the experiences. Carla and Franklin indicated that retirement housing should be culturally sensitive to both homosexual and heterosexual partners: It almost seems that I have a hard time picturing myself being treated differently than any other person, any other heterosexual person in a care facility... I think the things that we want are sensitive to us and our partners. If we're married or . . in any of those sorts of things, they are unique things we experience. (Carla) Healthcare workers need to be sensitive people and knowing what they are doing is a good thing ... they need to enhance support systems that effectively honour relationships and accommodate partners of LGB older adults.

(Franklin) Carla doesn't see herself as different from every other older adult in a healthcare facility but would want healthcare providers to honour all relationships and be culturally sensitive to LGB people and their partners. Carla believes that LGB older adults have unique experiences and understanding of thingsfrom work, marriage, relationships, social and life experiences, daily life routine, and all other sorts of things that play a significant role. Franklin wants healthcare providers to honour relationships and remain sensitive to LGB issues including the design and implementation of social programs for older adults. Franklin also wants a support system in place for older adults: help from blended families, inter-racial families, inter-religious families, same-sex headed families, single-parent families, best friends, and caregivers' sensitivity to LGB relationships and history. Having these support 
systems enables LGB older adults to achieve positive identity and resist stereotypes associated with gender roles and sexual identity classification. Franklin also believes there are fundamental questions that need to be asked before admitting any older adult into a facility. In his own words: "Is there somebody here that you already know, and you would want to be paired with? If you can't be in the same room, can you be in adjoining rooms?" These questions appeared to be powerful, allowing older adults their autonomy and right to self-determination. Also, these questions may address older adults' emotional and psychological issues. Autonomy and self-determination in this case enhances positive identity and the freedom of expression, such that older adults are able to display their masculine and feminine traits. Justin discussed some of his concerns:

I think we should be allowed to cohabit with our significant other, even if my partner was not requiring any services in the facility ... that's one of the most important things that I would want to see in retirement housing. I wouldn't want to be forced to part, given that I'm a gay man and even as a heterosexual person.

Justin echoes the desired intentions of LGB older adults: how they prefer to spend their entire life with their significant other. Sensitivity to these concerns may validate the wishes of the research participants. Leininger's theory may also address the issues of cultural insensitivity by promoting societal changes, optimal and holistic care, and guiding the practice of healthcare providers Leininger [4] his practice influences and/or helps LGB older adults to improve their human condition and deal with illnesses and other challenges. However, several questions may arise as to how older adults construct meaning and understanding out of their experiences and perceptions of the world. Here are some of the questions: Is it possible for older adults to cohabit in a care facility? Does this require a policy change? Who bears the associated cost of cohabiting in a care facility? Do older adults have enough income to do this? Can their pension cover this cost?

Study participants understood the implications of aging and the challenges that go along with retirement. As Abraham said, "I know we are different a bit ... I don't see much difference than anybody else growing older." Justin remarked, "You know, aging comes along with health issues and changes in your abilities." These quotes illustrate that older adults have a desire for mixed retirement facility. This acceptance and inclusiveness in retirement housing and services facility, raised through LGB older adults' interactions with the world, may also speak to their beliefs and value systems about aging. Knowledge and meaning for LGB older adults are generated from a combination of notions created in their minds and their life experiences. Leininger's theory in this regard empowers the researcher to support LGB older adults as they interface with the mainstream culture at retirement and intended to promote optimal care Leininger [6]. It is unclear whether young LGB adults share the same views and beliefs as older LGB older adults. Finally, the researcher believes that the mixed nature of existing retirement housing and service facility within BC Northern Health Authority boundaries further validates participants' understanding and meaning of retirement housing as previously understood. Also, this may imply encouragement in diversity of culture and inclusiveness in services. The next heading presents the discussion for this study

\section{Discussions}

As observed from the findings of this research, many LGB older adults referenced cultural sensitivity, honouring a relationship, and accommodating a partner as sensitive support needs that require attention in a care facility. LGB older adults have unique experiences and understandings of things. Work, marriage, relationships, social and life experiences, daily life routine-all these kinds of things matter and play a significant role Ghaziani [2]. Some participants believed that many healthcare workers are not culturally sensitive to these issues and, when brought to their attention, they remain unaddressed and/or treated as a low priority. Against this background, the application of Leininger's culture care theory ensures LGB older adults' values, beliefs, and holistic care are factored into their treatment plan, housing services, and housing needs. Holistic care implies that the healthcare worker becomes culturally sensitive and trained and can examine, acquire knowledge, interpret, and predict the phenomena in order to guide their practices. Cultural sensitivity may influence or help LGB older adults to improve their human conditions, address their housing needs, and deal with illnesses Leininger [4]. Holistic care is paramount since the majority of the participants in this research showed a great deal of interest in accommodating their partners in a care facility or retirement housing.

Participants strongly believed that older adults, whether in heterosexual or homosexual relationships, need not be separated from their partners and their loved ones, irrespective of their health conditions. This seemed to be a clear example of what Kimmel as cited in Knochel [35] postulated that LGB older adults face: the double stigmatization and oppression of both age and an unpopular sexual identity. However, validating older adults' feelings and honouring relationships, whether husband and wife, best friends or homosexual relationships, would empower older adults and promote autonomy to age successfully Jacobs \& Kane [36]. The perceptions and experiences of relationships as narrated by LGB older adults may open doors to the transformation and development of LGB older adults. This transformation and development starts with LGB older adults through social interactions within which certain cultural meanings are created, shared, and adopted Kiraly [37]. Therefore, knowledge is created as LGB older adults interact with their social environment, and in the process, both the LGB older adult and the environment are mutually changed Kiraly [37], Nonaka \& Von Krogh [38].

Some participants in this study believe that healthcare providers continually make assumptions about people's gender and sexual identity. Findings showed the need to engage and do a 
whole person assessment rather than basing sexual preferences on heterosexual assumptions. Beatrice, Edith, Marcus, and Justin would rather be asked if they have a husband than what their husband's name is, or who their family is than whether they have a family. Beatrice, Edith, Marcus, and Justin were very concerned with these heterosexual assumptions, attitudes, and unkind treatment from healthcare workers. Also, this research supports the notion that support systems/networks including help from blended families, inter-racial families, inter-religious families, same-sex headed families, single-parent families, best friends, and sensitive LGB caregivers are the best for LGB couples and families Hunter [9]. According to Fredriksen-Goldsen and Muraco [11], the perception of social and emotional support has a greater and more positive impact on the mental and physical health of the older adult than the actual support they receive. Social and healthcare providers should be consistently thinking of the best options to keep relationships together. Healthcare providers should keep the following questions in mind to achieve affirming and non-judgmental healthcare services: What services can we provide to keep relationships together? How do we support these relationships in a culturally sensitive manner? With these thoughts in mind, the social and healthcare needs of LGB older adults may be met.

\section{Recommendations}

Admitting this study area has no standardized social and healthcare services for LGB older adults. Some of the ways to improve the existing social and healthcare services include: providing cultural safety and training to employees and volunteers, providing affirming and non-judgmental services, acknowledging partners, and honoring relationships. This study is one of the first of its kind on LGB older adults within the study area. Further, this research is needed in order to have an in-depth knowledge and understanding of ageism as it affects LGB older adults.

Educating social and healthcare providers, caregivers and creating awareness with the general public about LGB cultural sensitivity, honouring a relationship, and accepting and accommodating a partner as sensitive support need to be discussed and addressed. Hospital leadership should create a more culturally sensitive intake / admission process. Such new admission protocols that acknowledge the social and healthcare needs of the LGB population should be supported at both federal and provincial levels. Refining intake and assessment processes will reduce the number of older adults on a waitlist seeking services and promote affirming and non-judgmental services that acknowledge partners and honour relationships. These efforts will have positive effects on the hospital bottom-line.

\section{Conclusion}

Social and healthcare professionals must actively work towards the advancement of knowledge and education about LGB issues and concerns. In this view, Leininger's culture care theory ensures LGB older adults' values, beliefs, and holistic care are factored into social and healthcare services received by this population. Holistic care implies that the social and healthcare worker becomes culturally sensitive and trained and can examine, acquire knowledge, interpret, and predict the phenomena in order to guide their practices. Further, Leininger's culture care theory ensure understanding and knowledge of the real world, assisting, supporting, or enabling LGB older adults with the intention of improving social and healthcare services and human conditions McFarland [39,40].

\section{References}

1. Canada Revenue Agency (2012) Changes to the Canada Pension Plan (CPP) for individuals who are 60 to 70 years of age.

2. Ghaziani A, Taylor V, Stone A (2016) Cycles of sameness and difference in LGBT social movements. Annual Review of Sociology 42: 165-183.

3. Leininger M (2006b) Ethnonursing: A research method with enablers to study the theory of culture care.

4. Leininger M (2006a) Culture care diversity and universality theory and evolution of the ethnonursing method.

5. Doornbos M, Zandee GL, DeGroot I (2014) Attending to communication and patterns of interaction: Culturally sensitive mental health care for groups of urban, ethnically diverse, impoverished, and underserved women. Journal of the American Psychiatric Nurses Association 20(4): 239-249.

6. Leininger M (1988) Leininger's theory of nursing: Cultural care diversity and universality. Nursing Science Quarterly 4: 152-160.

7. Christensen S (2014) Enhancing nurses' ability to care within the culture of incarceration. Journal of Transcultural Nursing 25(3): 223-231.

8. McFarland M, Wehbe Alamah H (2015) Leininger's culture care diversity and universality: A worldwide nursing theory ( $3^{\text {rd }}$ edn) Burlington MA: Jones \& Bartlett.

9. Hunter S (2013) Midlife and older LGBT adults: Knowledge and affirmative practice for the social services. New York.

10. Rosenfeld MJ (2010) Nontraditional families and childhood progress through school. The Demographics 47(3): 755-775.

11. Fredriksen Goldsen KI, Kim HJ, Barkan SE, Muraco A (2013) Health disparities among lesbian, gay, and bisexual older adults: Results from a population-based study. American Journal of Public Health 103(10): 1802-1809.

12. Gates GJ (2013) LGBT parenting in the United States. The Williams Institute.

13. Fredriksen Goldsen K, Muraco A (2010) Aging and sexual orientation: A 25-year review of the literature. Research on Aging 32(3): 372-413.

14. Pinsker DM, McFarland K, Pachana NA (2010) Exploitation in older adults: Social vulnerability and personal competence factors. Journal of Applied Gerontology 29(6): 740-761.

15. Taylor RJ, Chatters LM, Woodward AT, Brown E (2013) Racial and ethnic differences in extended family, friendship, fictive kin and congregational informal support networks. Interdisciplinary Journal of Applied Family Studies 62(4): 609-624.

16. Stone AL (2016) The impact of anti-gay politics on the LGBTQ movement. Sociology Compass 10(6): 459-467.

17. Ghaziani A, Baldassarri D (2011) Cultural anchors and the organization of differences: A multi-method analysis of LGBT marches on Washington. American Sociological Review 76(2): 179-206.

18. Almeida J, Johnson RM, Corliss HL, Molnar BE, Azrael D, et al. (2009) Emotional distress among LGBT youth: The influence of perceived discrimination based on sexual orientation. Journal of Youth and Adolescence 38(7): 1001-1014. 
19. Chance TF (2013) Going to pieces over LGBT health disparities: How an amended Affordable Care Act could cure the discrimination that ails the LGBT community. Journal of Health Care Law \& Policy 16(2): 375-402.

20. Gallicano TG (2014) Book review: Coming out of the closet: Exploring LGBT issues in strategic communication with theory and research. Journalism \& Mass Communication Quarterly 91(1): 192-195.

21. Statistics Canada (2017). Police-reported hate crime in Canada 2015.

22. Cohen J, Ezer T (2013) Human rights in patient care: A theoretical and practical framework. Journal of Health and Human Rights 15(2): 7-19.

23. Hoy Ellis CP (2016) Concealing concealment: The mediating role of internalized heterosexism in psychological distress among lesbian, gay, and bisexual older adults. Journal of Homosexuality 63(4): 487-506.

24. Ireland N (2015) Canadian forces sexualized culture hostile to women, LGBT member: Report. Ottawa Sun.

25. Rumens N, Broomfield J (2012) Gay men in the police: Identity disclosure and management issues. Human Resource Management Journal 22(3) 283-298.

26. Stanley IH, Duong J (2015) Mental health service use among lesbian, gay, and bisexual older adults. Psychiatric Services 66(7): 743-749.

27. Milner C, Van Norman K, Milner J (2012) The media's portrayal of ageing. Global population ageing: Peril or promise.

28. Wallace SP, Cochran SD, Durazo EM, Ford CL (2011) The health of aging lesbian, gay and bisexual adults in California PB2011-2: 1-8.

29. Creswell JW (2012) Qualitative inquiry and research design: Choosing among five approaches $3^{\text {rd }}$ edn. Thousand Oaks, CA: Sage.

30. Bryman A (2015) Social research methods $5^{\text {th }}$ edn New York, NY: Oxford University Press.
31. Boyatzis RE (1998) Transforming qualitative information: Thematic analysis and code development. Thousand Oaks CA: Sage.

32. Braun V, Clarke V (2006) Using thematic analysis in psychology. Qualitative Research in Psychology, 3(2): 77-101.

33. Meeks DW, Smith MW, Taylor L, Sittig DF, Scott JM, et al. (2014) An analysis of electronic health record-related patient safety concerns. Journal of the American Medical Informatics Association 21(6): 10531059.

34. Novak JD (2010) Learning, creating and using knowledge: Concept maps as facilitative tools in schools and corporations. New York, NY: Routledge.

35. Knochel KA, Quam JK, Croghan CF (2011) Are old lesbian and gay people well served? Understanding the perceptions, preparation, and experiences of aging services providers. Journal of Applied Gerontology $30(3): 370-389$

36. Jacobs RJ, Kane MN (2012) Correlates of loneliness in midlife and older gay and bisexual men. Journal of Gay \& Lesbian Social Services 24(1): 40-61.

37. Kiraly D (2014) A social constructivist approach to translator education: Empowerment from theory to practice. New York, NY: Routledge.

38. Nonaka I, Von Krogh G (2009) Perspective-tacit knowledge and knowledge conversion: Controversy and advancement in organizational knowledge creation theory. Organization Science 20(3): 635-652.

39. MM Leininger, MR McFarland. Culture care diversity and universality: A worldwide nursing theory ( $2^{\text {nd }}$ edn $)$, p. 1-42.

40. MM Leininger, MR McFarland. Culture care diversity and universality: A worldwide theory of nursing ( $\left.2^{\text {nd }} e d n\right)$, p. 43-82.

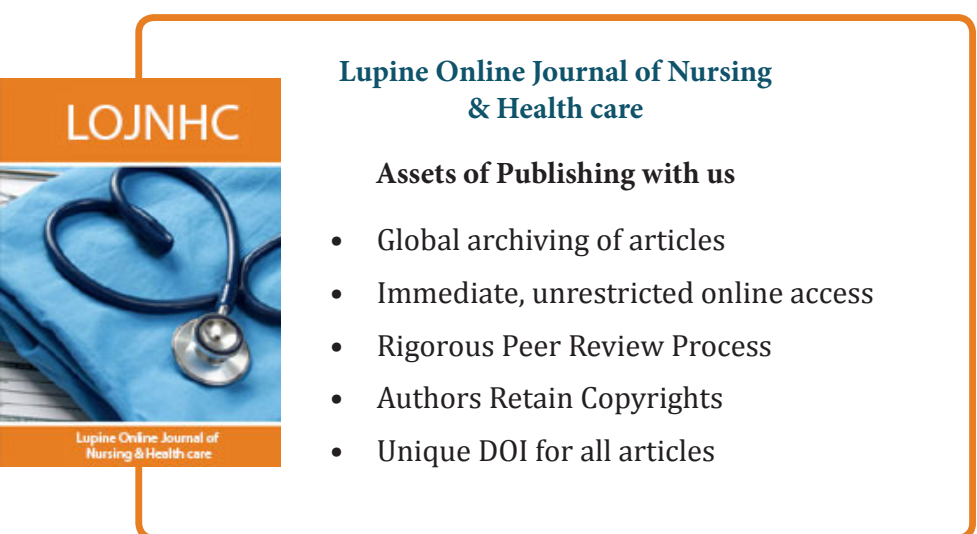

